\title{
Design of liquid detection sensor with low-frequency electromagnetic field
}

\begin{abstract}
This paper presents the design of liquid detection sensor with low-frequency electromagnetic field. The purpose of this sensor is to detect a change in the conductivity or permittivity of the liquid. If the evaluation frequency is less than $100 \mathrm{kHz}$, skin effects would be negligible. This paper proposes sensor that consists of two cylindrical sensors, a solenoid coil and a cylindrical capacitor. For an ideal shape condition, estimation methods for inductance and capacitance have been already proposed. However, the practical issues need to be clarified. It starts with fabrication of several coils and capacitors, to confirm the validity of the estimation methods. From experimental results, it was found that, the estimation error and existence of the parasitic element could not be neglected. This paper also presents a liquid detection demonstration with fabricated sensors based on differences in conductivity and permittivity.
\end{abstract}

Keyword: Cylindrical capacitor; Electromagnetic field; Liquid detection sensor; Solenoid coil 